\title{
SMART TRANSPORTATION APPLICATIONS FOR CITY BUSES
}

\author{
Serkan MEZARCIÖZ ${ }^{1}$, Enis AYTAR ${ }^{1}$, Murat DEMİRDÜZEN ${ }^{1}$, Mert \\ ÖZKAYNAK ${ }^{1}$, Kadir AYDIN ${ }^{2}$ \\ ${ }^{1}$ Temsa Global San. Ve Tic A.Ş. R\&D Center \\ ${ }^{2}$ University Of Cukurova, Department Of Automotive Engineering
}

\begin{abstract}
Passenger counting systems can help to fleet managers to plan route of city buses effectively by supplying the information of number of passenger inside a bus. By combining passenger counting system with a GPS system, location of the bus and number of passenger in the vehicle can be monitored online and an effective plan of city transportation can be done. With this system estimated time of arrival for the next station with the rate of fullness can be calculated and this information can be supplied to the fleet management center.
\end{abstract}

Application of IP (Internet Protocol) cameras will provide online information about the vehicle inside and surrounding to the fleet management center. If the vehicle deviates from its standard route or panic buttons located near driver and inside passenger cabin are pressed, an emergency signal can be sent to the fleet management center and vehicle can be monitored simultaneously via IP Cameras.

By checking the position of the vehicle via GPS system, advertisements or touristic information near the region can be displayed in special LCD monitors located between the glazes of side glasses.

In the current study, application details of these systems to a 12 meter low floor city bus, system components, and software details were discussed and explained in detail. 
2 Serkan MEZARCIÖZ, Enis AYTAR, Murat DEMIRDÜZEN, Mert ÖZKAYNAK, Kadir AYDIN

Keywords: Passenger counting system, GPS, smart city transportation, IP Camera, Panic Buttons, Internet of Thing

\section{INTRODUCTION}

Using electronic infrared beams or mechanical treadle mats, automatic passenger counters (APCs) have the ability to count transit passengers as they board and a light transit vehicles at individual stops. When coupled with stop location information, archived APC data can be post-processed to generate disaggregate data in both time and space. [1].

Embedded GPS receivers in vehicles' on-board units (OBUs, a common term for telematics devices) receive signals from several different satellites to calculate the device's (and thus the vehicle's) position. [2].

Smart transportation application for city buses consists of location based passenger counting system, safety package which includes IP cameras and panic buttons and location based advertisement system with special LCD monitors located between the glazes of side glasses.

Passenger counting systems can help to fleet managers to plan route of city buses effectively by supplying the information of number of passenger inside a bus. By combining passenger counting system with a GPS system, location of the bus and number of passenger in the vehicle can be monitored online and an effective plan of city transportation can be done. With this system estimated time of arrival for the next station with the rate of fullness can be calculated and this information can be supplied to the fleet management center. 


\section{MATERIAL AND METHOD}

Passenger counting system consist of passenger counting unit, 8 infrared sensors, GPS antenna, GSM antenna, Ethernet connection and vehicle parameters as shown below in Figure 1. The vehicle has a handrail in the middle of front door, so 2 infrared sensors are enough for the front door to obtain high accuracy while 3 infrared sensors are used at middle and rear door. All sensors are connected to the passenger counting unit. PCU works with $24 \mathrm{~V}$ DC power and all sensors take power from PCU. GPS antenna is connected for location information, GSM antenna and Ethernet connection is for data transfer. PCU also needs some vehicle parameters such as ignition status, door status for each door separately and vehicle speed information. We make necessary adjustment on PCU such as FTP server address, port, user name, password etc. Then, PCU starts to send data to the server.

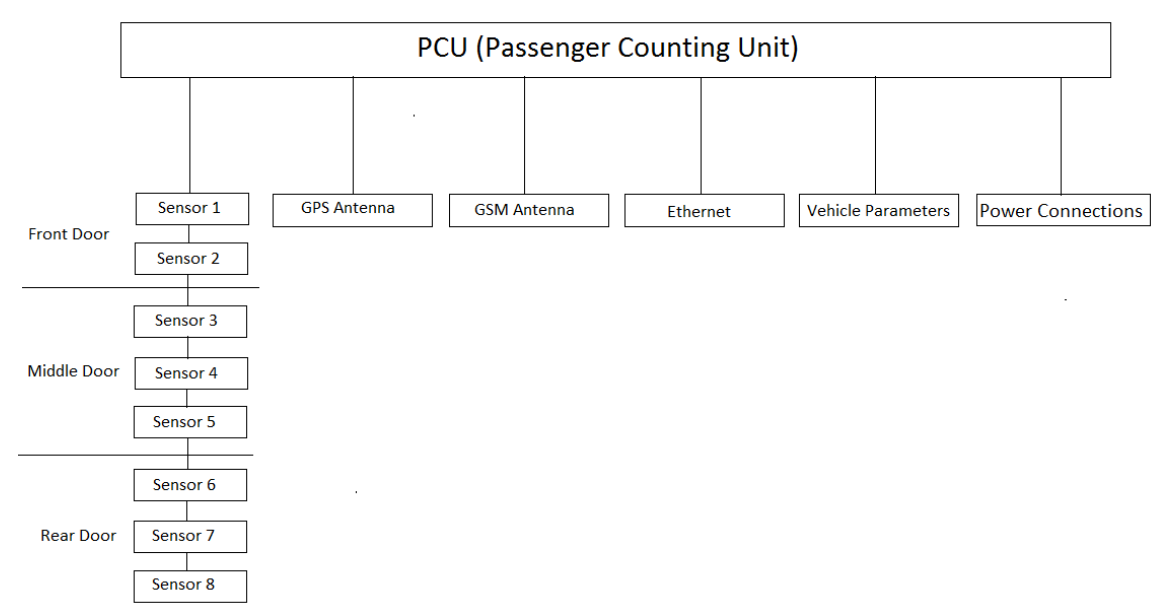

Fig. 1. General overview of passenger counting system

By this study, municipalities and fleet management center can have location of the bus, time date information and number of passenger in the vehicle. According to these parameters they can make an effective plan of city transportation. For example, when they analyze 
these parameters, they can observe that between 08:00 am and 10:00 am the vehicle has always a high fullness rate even overloaded. They can assign a bigger vehicle $(12 \mathrm{~m}$ vehicle instead of $9 \mathrm{~m})$ or additional vehicles for such high fullness rate hours. Also, they can analyze if expected travel time and real travel time for the routes are fitted or not. They can define different travel time according to fullness rate and traffic jam.

Safety package of the system includes IP camera and panic button system which consist of IP cameras, poe switch, network video recorder, solid state drive (SSD), 7" touchable monitor, GPS antenna, GSM antenna, panic buttons and power connections as shown below in Figure 2. 3MP IP camera with its cover is used for rear view, other 3MP IP cameras are used for front view, driver area and third door area. 5MP IP camera which can record $360^{\circ}$ is used for inside of the vehicle and mounted middle of the vehicle. All cameras are directly connected to the POE switch via RJ45 cables. POE switch is connected to the network video recorder via RJ45 cable. Network video recorder save recordings in its hard disk, display for driver via 7" touchable monitor and send to server via GSM antenna. Panic buttons directly connected to the network video recorder and network video recorder send emergency signal and location information whenever one of panic buttons is pressed via GPS and GSM antennas. Network video recorder can work with a power of 9-36V DC. 


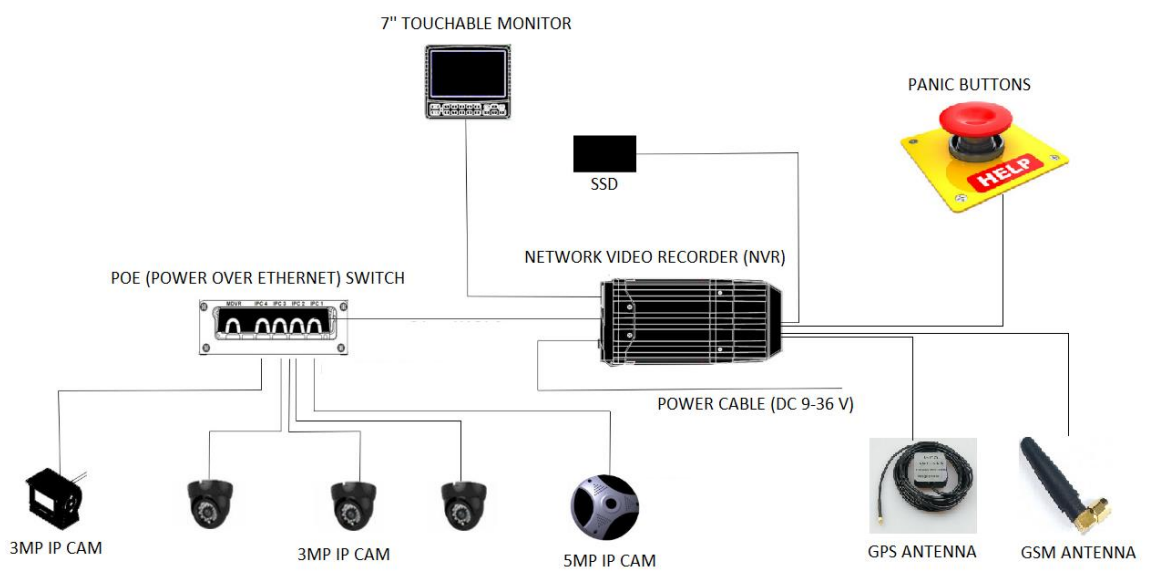

Fig. 2. General overview of safety package

Application of IP (Internet Protocol) cameras will provide online information about the vehicle inside and surrounding to the fleet management center. If the vehicle deviates from its standard route or if any insecure situation occurs during the travel, driver or passengers can press panic buttons which are located near driver area and inside passenger cabin. Then, an emergency signal and location information can be sent to the fleet management center and vehicle can be monitored simultaneously via IP cameras. With the application of IP cameras, drivers know that every behavior of them is recorded so they push themselves to obey traffic rules and passenger safety strictly.

Location based advertisement system consists of Linux PC, GPS, GPRS and power source as shown below in Figure 3. Linux PC decides to which advertisement will be displayed at screens by comparing location information which comes from GPS and previously defined location information in its software. Any advertisement, video or picture can be displayed. GPRS module is used to be able to update Linux PC's software and advertisement. Power source is used to supply power to all system. Its input voltage range is between $5 \mathrm{~V}$ and $38 \mathrm{~V}$. Its output voltage is $5 \mathrm{~V}$ and supply power to all other components. 


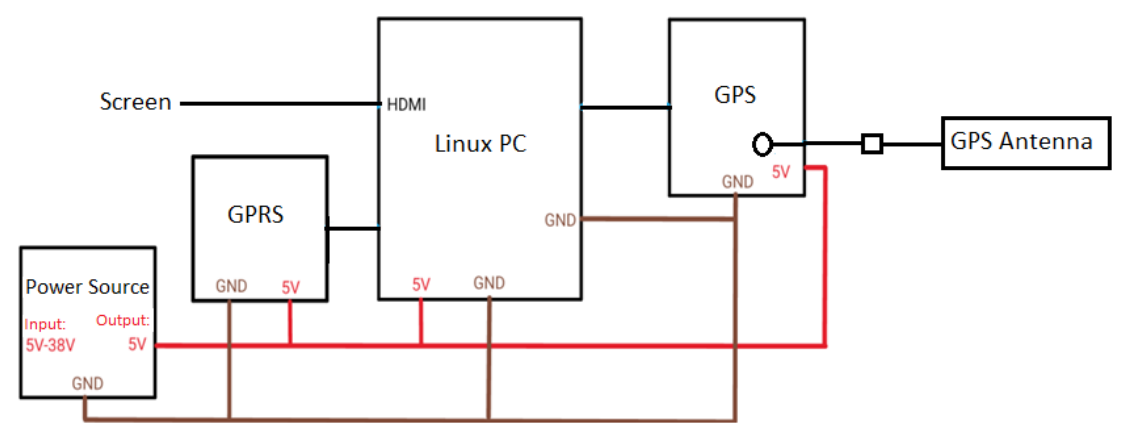

Fig. 3. General overview of location based advertisement system

We present to have a chance to be able to display different advertisements with only one system to municipalities, owners or fleet managers instead of a constant and unique advertisement. They can display advertisements about nearest hotels, restaurants, shopping centers, touristic areas and historical places according to vehicles location information. They can display information about local government if desired. Also, we present them to have a chance to update their advertisement via USB or internet quickly.

Another feature of this application is LCD monitors located between the glazes of side glasses. Municipalities, owners or fleet managers can be able to select LCD monitors located between the glazes of side glasses instead of standard 19" monitor in vehicles. The risk of crashing to monitors by passengers and breakdown of monitors by external effects disappear by LCD monitors located between the glazes of side glasses. The system is compatible with both LCD monitors located between the glazes of side glasses and standard 19" monitors.

\section{CONCLUSSION}

We expect to present an effective routing plan to municipalities, owners and fleet managers by location based passenger counting system. Municipalities, owners and fleet managers can make an effective plan of city transportation according to location, time and 
fullness rate information. City transportation will be safer with the application of safety package which includes IP cameras and panic buttons. Drivers know that every behavior of them is recorded so they push themselves to obey traffic rules and passenger safety strictly. We present to municipalities, owners and fleet managers to have a chance to update their advertisement via USB or internet quickly and without any additional cost by location based advertisement system. Municipalities, owners and fleet managers don't have to display constant and unique advertisement at exterior surface of the vehicle as it is up to now. In this way, their advertisement income increases significantly.

\section{REFERENCES}

[1]. CUTR, 2010, A Guidebook for Using Automatic Passenger Counter Data for National Transit Database (NTD) Reporting, National Center for Transit Research, University of South Florida, pp. 58.

[2]. Ezell, S.,2010, Intelligent Transportation Systems, The Information Technology \&Innovation Foundation, pp.58 